\title{
La política exterior de Tailandia y su participación en la ASEAN, el ARF y el APEC
}

$\mathrm{L}$ a participación de Tailandia en los organismos de cooperación de la Cuenca del Pacífico ha sido muy activa, aunque concentrada en los que tienen una cobertura más localizada en el sudeste de Asia. En el presente trabajo se abordará de forma general la manera en que este país concibe sus relaciones con otros países, principalmente de la región, y cómo con base en sus expectativas se define su actuación en los diferentes foros regionales.

Las condiciones resultantes de la segunda guerra mundial fueron turbulentas, sobre todo para los países del sudeste de Asia, quienes sufrieron de tensiones internas que frecuentemente terminaron en fuertes enfrentamientos civiles con sus consecuencias sangrientas la mayoría de las ocasiones. Si bien estos enfrentamientos fueron por lo general en los países recién independizados, además hubo fricciones entre estas naciones como, por ejemplo, el episodio suscitado entre Singapur y los territorios de Sabah y Sarawak (ahora parte de Malasia), dominados entonces por Gran Bretaña. Lo anterior motivó que Tailandia viera la necesidad de establecer relaciones más fuertes con los nuevos líderes de sus vecinos surgidos de movimientos de independencia recientes, todo esto en un marco en el que se conjuntaba también el fin de una época colonial en la región, la guerra en Vietnam y la creciente amenaza comunista.

Con el paso del tiempo el ambiente político fue mejorando y algunos acontecimientos en la región dieron pie a que las tensiones disminuyeran. Por ejemplo, en Indonesia el nuevo orden, representado entonces por el presidente Suharto, puso fin a la confrontación con Malasia; en Filipinas otro cambio de gobierno

* Investigador del Departamento de Estudios del Pacífico de la Universidad de Guadalajara.

ORCID http://orcid.org/0000-0003-0344-6895 trajo una mejora de las relaciones existentes entre estos dos últimos, eventos en los cuales Tailandia jugó el importante papel de conciliador. No obstante, la situación no podría ser juzgada de excelente, pues en el resto de la región, principalmente Indochina (es decir, Camboya, Laos y Vietnam), la amenaza comunista seguía latente.

De esta manera, la política exterior de Tailandia se vio caracterizada por un enfoque anticomunista y sumamente influenciada por Estados Unidos. Esto también en función de que los grupos militaristas eran los más fuertes en la toma de decisiones políticas y su alineación con Estados Unidos era de conveniencia mutua. Así, entre más estrecha era la relación de Tailandia con Estados Unidos, más difícil se tornaba la propia relación con sus vecinos de Indochina, por lo que la situación era de suma hostilidad. ${ }^{1}$ Pero con el resto de los vecinos de la región, en especial los que más coincidían con el enfoque anticomunista, se tenían buenos lazos diplomáticos, lo que condujo a la exploración de áreas de congruencia y convergencia en sus aspiraciones. Esto culminó en la histórica reunión de Bangkok, Tailandia, el 8 de agosto de 1967, a la que asistieron los ministros de relaciones exteriores de Indonesia, Filipinas, Malasia, Singapur y Tailandia, en la que se constituyó la Asociación de Naciones del Sudeste de Asia (ASEAN) mediante la firma de la Declaración de la ASEAN (también conocida como Declaración de Bangkok).

Un ambiente diferente se empezó a percibir una vez que Estados Unidos abandonó Vietnam a principios de los setenta. De este modo, hacia mediados de 1976, después de una intensa 'ofensiva' diplomática, se produjo el restablecimiento de relaciones diplomáticas con Camboya y Vietnam (con Laos habían permanecido), iniciando así una nueva, aunque 
corta, etapa en las relaciones con Indochina, lo que representó un cambio drástico del idealismo al pragmatismo en la concepción de la política exterior de Tailandia. Sin embargo, el golpe de Estado perpetrado a principios de octubre de 1976 por los militares al gobierno liberal de Seni Pramoj, también asestó un duro golpe a la reciente reconciliación con Indochina, la cual empeoró hacia finales de 1978, debido a la invasión de Camboya por parte de Vietnam. ${ }^{2}$

No fue sino hasta 1988 cuando las tensiones entre Tailandia e Indochina, particularmente Vietnam, empezaron a mejorar, una vez que las tropas vietnamitas empezaron a desalojar Camboya. Además, en agosto de 1988 se nombró a Chatichai Choonhavan como primer ministro, quien dio un giro a la política exterior de Tailandia, desde la concepción de la diplomacia política hacia la concepción de la diplomacia económica. Así, una de sus primeras iniciativas fue la de "transformar a Indochina de un campo de batalla a un mercado". ${ }^{3}$ Con esto Chatichai estaba inaugurando una nueva etapa en las relaciones exteriores de Tailandia, con las cuales se trataría de fomentar un mayor grado de flexibilidad en las relaciones comerciales con el exterior, particularmente con Indochina.

A pesar de que Chatichai fue destituido por un golpe de Estado en febrero de 1991, la orientación de la política de relaciones exteriores ha seguido, en términos generales, hasta la actualidad el enfoque propuesto por su administración. De modo que el primero de los ejes conductores de la actual diplomacia de Tailandia se propone "llevar la política exterior haciendo hincapié en la diplomacia económica proactiva, así como otras formas de diplomacia con la visión de restaurar y estrechar las relaciones internacionales de Tailandia, además de buscar la cooperación en todos los campos". ${ }^{4}$
De esta manera, la participación de Tailandia en la ASEAN está estrechamente ligada a las expectativas de este país, dependiendo de la etapa en la que se encontrara y de acuerdo con sus intereses particulares. Así, cuando la ASEAN fue formada, en 1967, eran tiempos difíciles para la región, lo cual despertó gran escepticismo sobre su supervivencia. Sin embargo, con el paso del tiempo, y no sin grandes esfuerzos por parte de sus miembros, ésta se convirtió en un actor clave no solo en la región del sudeste de Asia sino en todo Asia Pacífico. Tailandia, además de haber sido un factor importante para la formación de la ASEAN, también jugó un papel fundamental en el aspecto político, el cual fue uno de los principales objetivos en el establecimiento de la misma, ya que los líderes tailandeses, en especial el entonces ministro de relaciones exteriores, Thanat Khoman, reconocieron la urgente necesidad de reunirse en alguna forma de agrupación con fines de cooperación con miras a la promoción de la paz y la estabilidad en la región, así como trabajar en busca del beneficio conjunto.

Por esta razón y por ser el país miembro más expuesto a las eventualidades en la zona, durante mucho tiempo la política seguida por la ASEAN hacia Indochina estuvo marcada por la política de relaciones exteriores de Tailandia. Por ejemplo, el boicot económico y político de la ASEAN contra Camboya y Vietnam estuvo encabezado por Tailandia. Además, la invasión de Camboya por parte de Vietnam y la instalación de la República Popular de Kampuchea (Camboya) recrudecieron aun más la hostilidad hacia Indochina, razón por la que Tailandia movilizó a los miembros de la ASEAN para pedir el apoyo de su postura al respecto y propuso una fuerte oposición a dicha invasión. Así, durante los trece años de la ocupación vietnamita de Camboya, Tailandia 
prácticamente definió la agenda para la totalidad de los países de la ASEAN, así como de la asociación misma, con respecto a la política a seguir hacia Vietnam. ${ }^{5}$

Sin embargo, el drástico cambio sobre el enfoque de la política exterior hacia Indochina, diseñado por el primer ministro Chatichai, marcó un punto de partida en la relación diplomática. Esto tomó por sorpresa a la ASEAN, pues significó un cambio radical en la política seguida por la asociación hacia Indochina, tanto que sorprendió al propio ministro de relaciones exteriores de Tailandia, Siddhi Savetsila, y causó consternación entre el resto de los miembros de la ASEAN. De inmediato los ministros de relaciones exteriores de la asociación se pusieron en contacto con Siddhi Savetsila, quien prefirió en ese momento continuar con la política de línea dura hacia Vietnam y Camboya.

Chatichai, tratando de justificar la racionalidad económica de su política de relaciones exteriores, expresó que la política tomaría un segundo lugar, después de la economía, y que el bienestar económico sería una garantía de la seguridad de largo plazo. Además, asentó que el restablecimiento de relaciones diplomáticas con Vietnam era una de sus más altas prioridades y que Indochina debería ser transformada de una zona de guerra a una zona de paz, ligada con el sudeste de Asia a través de lazos comerciales, la inversión y comunicaciones modernas.

Ésta fue una visión que no cambió sustancialmente en los años siguientes, a pesar de los cambios de administración que se sucedieron. La ASEAN pronto siguió el ejemplo de Tailandia al respecto, sobre todo después de la salida de Vietnam de Camboya (en 1989) y de la firma del Acuerdo de Paz de París sobre el establecimiento de la paz en Camboya (en 1991), y los países miembros adoptaron su propia agenda con respecto a Vietnam. Esto trajo una cierta normalización en las relaciones diplomáticas entre la ASEAN e Indochina, que se reflejó en la admisión de Vietnam como miembro de pleno derecho en la asociación el 28 de julio de 1995. Camboya y Laos, junto con Myanmar, se incorporarían en 1997, pero sólo se admitió a los dos últimos y se pospuso la admisión de Camboya, la que finalmente se llevó a cabo el 30 de abril de 1999.

Tailandia también ha desempeñado un papel relevante en otras áreas de actividad de la ASEAN. Participó activamente en la propuesta para la declaración de la ASEAN como la zona de paz, libertad y neutralidad (ZOPFAN), firmada el 27 de noviembre de 1971 en Kuala Lumpur, Malasia; trabajó, asimismo, en la preparación del Tratado de Amistad y Cooperación (TAC), firmado durante la primera reunión cumbre realizada el 24 de febrero de 1976 en Bali, Indonesia, y que junto con la Declaración de Bangkok constituyen los pilares básicos que guían los principios y objetivos de la ASEAN. Además, Tailandia y Filipinas propusieron la creación del Foro Regional de la ASEAN (ARF), del que hablaremos posteriormente, y el Tratado sobre la Zona Libre de Armas Nucleares del Sudeste de Asia (SEANWFZ). Como un reconocimiento a su labor en estos esfuerzos, Tailandia fue nombrado depositario de los tratados mencionados.

Con la evolución de la ASEAN, ésta ha puesto cada vez más atención a los temas económicos, como inversión y comercio, los cuales se han constituido en parte fundamental de su funcionamiento, sobre todo después de terminada la guerra fría. Así, con el fin de extender la cooperación de la ASEAN en asuntos económicos, el entonces primer ministro de Tailandia, Anand Panyarachun, propuso la creación del Área de Libre Comercio de la ASEAN (AFTA), que fue aprobado por los jefes de Estado en la Cuarta Reunión Cumbre de 1992, en Singapur. Asimismo, otras áreas de cooperación económica han emergido, como por ejemplo el Esquema de Cooperación Industrial de la ASEAN (AICO).

Por otra parte, en la Quinta Reunión Cumbre celebrada en Bangkok, Tailandia, en diciembre de 1995, la cooperación social y funcional fue elevada a un plano superior, de manera que el tema de la Declaración de 
Bangkok (de 1995) fue: Prosperidad compartida por medio del desarrollo humano, la competitividad tecnológica y la cohesión social. En esa ocasión se creó la Fundación ASEAN, con el fin de apoyar actividades educativas y de intercambio, cuya base la constituyó un fondo de desarrollo social del entonces ministro del exterior tailandés Amnuay Virawan.

Tailandia desempeñó también un papel importante en la adopción de la Visión de la ASEAN 2020 durante la Segunda Reunión Cumbre Informal, en diciembre de 1997, la cual se propone servir de guía a la asociación en el siglo XXI. Asimismo, en la Sexta Reunión Cumbre, llevada a cabo en Hanoi, Vietnam, en diciembre de 1998, Tailandia presionó para alcanzar un acuerdo que reforzara los esquemas existentes, y la creación de nuevos, con el fin de hacer frente de manera más exitosa a la crisis regional. Así, contempló la necesidad de hacer hincapié en el desarrollo de los recursos humanos, la eliminación de la pobreza y la disminución de las desigualdades socioeconómicas, la tecnología de la información e infraestructura, el desarrollo sustentable y el ambiente, y la disminución del impacto social de la crisis financiera que azotaba a la región.

Por lo que toca a la participación de Tailandia en el ARF, tenemos que fue uno de los iniciadores de la idea de crear un cuerpo consultivo con miras a reforzar la confianza entre los países con intereses sobre la seguridad en la zona. Fue Tailandia quien en 1990 organizó, junto con Filipinas, una serie de dos seminarios sobre seguridad regional en Asia Pacífico, con un enfoque especial sobre el sudeste de Asia. La contribución de estos seminarios fue incorporar el tema de la seguridad regional en la agenda de la ASEAN y en varios de sus diálogos institucionales. Así, en la Cuarta Reunión Cumbre de 1992 en Singapur, la primera reunión de los líderes de la ASEAN después del fin de la guerra fría, se decidió promover el diálogo externo e interno con el fin de fomentar la seguridad en la región, para lo que se inició una serie de foros tendentes a discutir la manera en que se pudiera reforzar la cooperación en asuntos de seguridad regional.
De esta manera, el 25 de julio de 1994 se llevó a cabo la primera reunión del ARF en Bangkok, Tailandia. Desde entonces, el ARF ha tenido un progreso satisfactorio, de acuerdo con sus objetivos, de cooperación en seguridad, donde participan los ministros del exterior y oficiales de defensa, por lo que ha sido capaz de abordar temas de importancia significativa, incluyendo las pruebas nucleares en el sur de Asia y el impacto de la crisis económica y financiera sobre la seguridad regional.

Las actividades de las reuniones ministeriales anuales del ARF se han expandido también a diferentes áreas, como la prevención de desastres, búsqueda y rescates y el mantenimiento de la paz. En estas áreas Tailandia y Nueva Zelanda dirigieron mancomunadamente las reuniones del ARF sobre prevención de desastres durante 19971998; junto con Estados Unidos, durante 19981999 coordinó las actividades llevadas a cabo en el marco del grupo sobre medidas para el fomento de la confianza y, en conjunción con la Unión Europea, durante el mismo período anterior coordinó los trabajos del taller sobre los enfoques del entrenamiento para el mantenimiento de la paz. Además, el ARF está evolucionando hacia la discusión de la interconexión entre las medidas para el fomento de la confianza y la diplomacia preventiva, área en la que Tailandia juega un papel de suma relevancia.

Ahora bien, Tailandia participa también en otros organismos de cooperación de la Cuenca del Pacífico, como el mecanismo de Cooperación Económica para Asia y el Pacífico (APEC), el Consejo Económico de la Cuenca del Pacífico (PBEC), el Consejo de Cooperación Económica del Pacífico (PECC) y la Conferencia sobre Comercio y Desarrollo del Pacífico (PAFTAD). Sin embargo, es en el mecanismo de APEC en donde se reflejan, en una gran proporción, las propuestas que se hacen por parte de los demás organismos de cooperación. De aquí que sea de gran interés el hecho de que vaya a ser Tailandia quien guiará el mecanismo de APEC durante el año 2003, justamente después de México, quien conducirá los trabajos durante 2002. 
Tailandia fue uno de los doce países que pusieron en operación el mecanismo de la APEC en noviembre de 1989, junto con Australia, Brunei, Canadá, Corea del Sur, Estados Unidos, Filipinas, Indonesia, Japón, Malasia, Nueva Zelanda y Singapur.

Por otra parte, fue durante la XI Reunión Ministerial efectuada durante septiembre de 1999 en Auckland, Nueva Zelanda, que se aceptó por parte de los ministros del APEC que Tailandia fuera la economía del grupo que encabezara la XV Reunión Ministerial a celebrarse durante el año 2003. Ya antes, en 1992, Tailandia había organizado la IV Reunión Ministerial, pero nunca ha organizado una reunión de líderes, puesto que éstas se iniciaron a partir de la reunión de Seattle, Estados Unidos, en 1993. Ahora Tailandia tendrá la oportunidad de, además de definir el curso del APEC durante 2003, figurar como la economía miembro responsable de la organización de la XV Reunión Ministerial y la XI Reunión de Líderes del APEC, las de más alto nivel del organismo. ${ }^{6}$

En suma, Tailandia participa en los diferentes organismos y foros de cooperación regional en el área de Asia y el Pacífico. De modo que "el nuevo gobierno de Tailandia quiere pensar y actuar más localmente, conduciendo la política exterior de un modo más 'asiático' ". ${ }^{7}$ Esto es posible dada la nueva situación en la región y la percepción de un ambiente más propicio para enfocar la política exterior desde otro ángulo, que responda más a intereses de tipo económico, puesto que las cuestiones políticas y de seguridad regional son ahora mantenidas en un nivel de mayor confiabilidad. Lo anterior gracias también al funcionamiento de los organismos de cooperación, sobre todo de la ASEAN y el ARF, que han contribuido a desarrollar un ambiente de estabilidad regional.

\section{Notas}

1 Surin Maisrikrod, "Thailand's Policy Dilemmas Towards Indochina", Contemporary Southeast Asia, vol. 14, núm. 3, diciembre de 1992, p. 291.

2 Ibid., p. 294.

3 Paisal Sricharatchanya, apud, Marc Innes-Brown y Mark J. Valencia, "Thailand's Resource Diplomacy in Indochina and Myanmar", Contemporary Southeast Asia, vol. 14, núm. 4, marzo de 1993, p. 333.

4 Policy of the Government of H. E. Prime Minister Thaksin Shinawatra Delivered to the National Assembly on Monday, 26 Februay 2001, en http:// www.thaigov.go.th/general/policy/policy.htm

5 Surin Maisrikrod, op. cit., p. 298.

6 APEC 2003 Work Plan, en http://www.APEC2003.org/ WorkPlan/workplan.html. También véase este sitio para una mayor precisión en cuanto al calendario de actividades propuesto.

7 "Back to Business", Far Eastern Economic Review, 22 de febrero de 2001, p. 26. Ty 